\title{
METHOD FOR MEASURING MOISTURE OF GRAIN MASS UNDER VIBRATION
}

\author{
Alexander Ivashina, Andrey Adoshev, Sergey Antonov, Elena Logacheva
}

Stavropol State Agrarian University, Russia

av_ivashina49@mail.ru, adoshev@mail.ru, antonov_serg@mail.ru, elena.logacheva2010@yandex.ru

\begin{abstract}
A detailed analysis of the end-to-end conductivity was carried out using experimental data from the measurement scheme, presented in the article. The oscillograms of changes in the time of the specific active conductivity of wheat grains of the given moisture at a given amplitude of vibration (on a direct current) were removed. Experiments have shown, that specific active conductivity varies from a value corresponding to a lack of vibration to a corresponding value, subject to vibration, i.e., almost more than 3 times. The minimum value of end-to-end conductivity when exposed to vibration, as shown by the experimental test, is almost an order of magnitude less than its average value. The decrease in the value of end-to-end conductivity relative to its average value is manifested in the intervals of time corresponding to the moments of movement of the sensor downwards, that is, in the direction of acceleration of gravity, under the influence of a vibrator. The work offers a way to measure the moisture of the grain mass and the device for its implementation. A distinctive feature of the developed method is that the controlled grain mass is filled in the cavity of the capacitive sensor and subjected to vertical vibration. The registration of dielectric permeability (moisture) is made at intervals of time corresponding to the minimum value of end-to-end conductivity. The proposed method makes it possible to significantly increase the accuracy of measuring the moisture content of the grain mass. This is especially important in conditions of high moisture.
\end{abstract}

Keywords: moisture, grain, vibration, dielectric permeability, conductivity.

\section{Introduction}

The electrical characteristics of grains and seeds are studied by many authors [1-12]. The issues of the influence of the vibration frequency on the reach-hrough conductivity of the vibro-fluidized grain mass have not been investigated.

In modern devices for controlling the moisture content of bulk materials, the achievement of reliable and reproducible results is impossible without special sample preparation. Sample preparation is reduced to maintaining its constant density over the entire range of controlled moisture $[1 ; 6]$ and is one of the most important tasks of sample formation for moisture control devices used in flows of various bulk materials.

A relatively simple and acceptable way to stabilize the density of a bulk material sample, as an interfering factor, is its positive seal, or spill with a free gravity outflow.

There are sample preparation devices with free (gravity) outflow of material. The test results of such devices showed that they can be recommended for steady flows of grain and granulated materials [2-5], but are unsuitable for fines, for example flour, which tend to stick to electrodes.

Flow devices have an excessive number of electrodes that deteriorate the operation of the primary converters, since the smoothness of the material update is impaired. In addition, fasteners are located in the cavity of the sample preparation device and disrupt the smoothness of the grain massflow, forming floating voids.

One of the main disadvantages of flow devices with gravity outflow of bulk materials is the zeroing of the moisture indicator during short interruptions or significant inhomogeneityin the flow of bulk materials, which leads to malfunctions of automatic moisture controllers and complicates the work of the operating personnel of grain dryers [12]. The devices can be used to measure moisture during drying in carousel and shaft type dryers and others when harvesting and processing grain crops at enterprises of the agro-industrial complex and of the food industry.

Finely dispersed bulk materials with a polydisperse particle size distribution, when vibrated, are divided into classes by size and are not protected against significant sticking. These factors impose significant restrictions on the use of such devices.

However, such devices are used for materials of monodisperse composition [6-9], for example, granulated mineral fertilizers, grain, etc.

Sample preparation devices, spilled with a power sealing arrangement, are divided into discontinuous devices, screw and roller devices [10-12]. The disadvantages of such a device include 
the need to use a rotating electric drive. Short interruptions in the supply of bulk materials lead to zeroing of the indicator of the measuring converter.

The screw type sample preparation devices combine the function of moving and sealing and are structurally made in the form of a short screw containing several turns. When the bulk density of the feed changes, its pressure on the walls of the sealing nozzle changes, and a signal appears in the strain gages that controls the actuator that moves the nozzle. The device is complicated in design, has a rotating electric drive and is practically unsuitable for controlling grain moisture, as it will lead to injury.

Self-packingsample preparation devices include those that are installed in chutes, surge bin, etc. The primary converter is mounted motionless and is rotated by its working plane at an angle to the horizontal symbol. The disadvantages of such devices include the possibility of their use only for technological purposes, when strict continuity of the incident stream is ensured.

Vibration action significantly reduces these forces, allowing the grains to fit more densely. The laying density depends on the vibration parameters $[6 ; 12]$.

The use of vibrational fluidizationfor controlling the moisture content of the grain mass allows to solve two problems at once, namely, stabilization of bulk density with the simultaneous export of bulk material through the sensor cavity. The article proposes a method for measuring the dielectric permeability (moisture) of the vibro-fluidized grain mass, increasing the accuracy of its measurement.

\section{Materials and methods}

The experimental results described in [12] have shown that the value of the dielectric permeability (DP) of the grain mass (GM) in the vibro-fluidized layer decreases, starting with some values of the vibration amplitude with the growth of the latter.

The experimental determination of the dependences of the specific reach-through conductivity of GM at a direct current under vibration and without it has been carried out according to the diagram presented in [12] in Fig. 1 and 2.

The oscillograms of changes in time of the specific active conductivity of "Bezostaya-1" wheat grain with a moisture content of $W=17 \%$ with a vibration amplitude of $A=1 \mathrm{~mm}$, are presented in Fig. 1.

As it can be seen from the oscillograms, thereach-through conductivity (at direct current) varies from a value of $\gamma_{0}$, corresponding to the absence of vibration, to anaverage value of $\gamma_{\text {avg }}$, corresponding to the effect of vibration, which is more than 3 times. The minimum value of the reach-through conductivity $\gamma_{\min }$ under vibration, as has been shown by experimental verification, is almost an order of magnitude less than its average value $\gamma_{\text {avg. }}$. A decrease in the value of the reach-through conductivity relative to its average value $\gamma_{\text {avg }}$ is manifested in time intervals corresponding to the moments of the downward movement of the sensor, that is, in the direction of gravity acceleration, under the action of a vibrator. Obviously, this phenomenon of change $\gamma$ can be explained by the fact that during the movement of the bulk material upward, that is, against the direction of gravity, its compaction occurs, and downward - loosening. Particles of bulk material in this phase of motion are in free fall, and contact between them decreases. The oscillograms similar to those shown in Fig. 1 have been obtained for grain and other crops.

The oscillogram shows that under vibration, the value of the minimum instantaneous reachthrough conductivity is 7.2 times less than its average value and almost 20 times less than the value of the reach-through conductivity in the absence of vibration. Thus, it is necessary to make the measurement of the informative parameter, dielectric permeability (DP) at the moment when the reach-through conductivity reaches the minimum values. This will significantly increase the accuracy of the measurement.

A distinctive feature of the developed method is that the controlled GM is poured into the cavity of the capacitive sensor and subjected to vertical vibration. The DP registration is performed at time intervals corresponding to the minimum value of the reach-through conductivity. The effectiveness of using this method will largely depend on the simplicity and reliability of the device, which 
automatically determines the time intervals for measuring moisture, during which the reach-through conductivity of the bulk material reaches its minimum values.

A diagram of the device that implements the proposed method for measuring the electrical permeability of the grain mass is presented in Fig. 2.

The device contains the half-wave voltage source 1, the output of which is connected to a serially connected coil of the electromagnetic vibrator 2 and the resistor 3. The electromagnetic vibrator 2 mechanically connected to the capacitive sensor 4, fixed on elastic springs 5 and filled with bulk material. In the sensor cavity 4 capacitor plates are installed 6 , connected to a secondary converter 8 . Resistor clamps 3 connected to the input of the threshold key 7, the input of which is connected to the controlled input of the secondary converter 8 . The converter output 8 is connected to the measuring device 9.

The device operates as follows. A low-frequency half-wave voltage of $50 \mathrm{~Hz}$ is supplied from the source 1 to the series-connected coil of the electromagnetic vibrator 2 and the resistor 3. Bulk material placed in the capacitive sensor 4 , fixed on elastic springs 5 , is exposed to vertical vibration created by the electromagnetic vibrator 2.

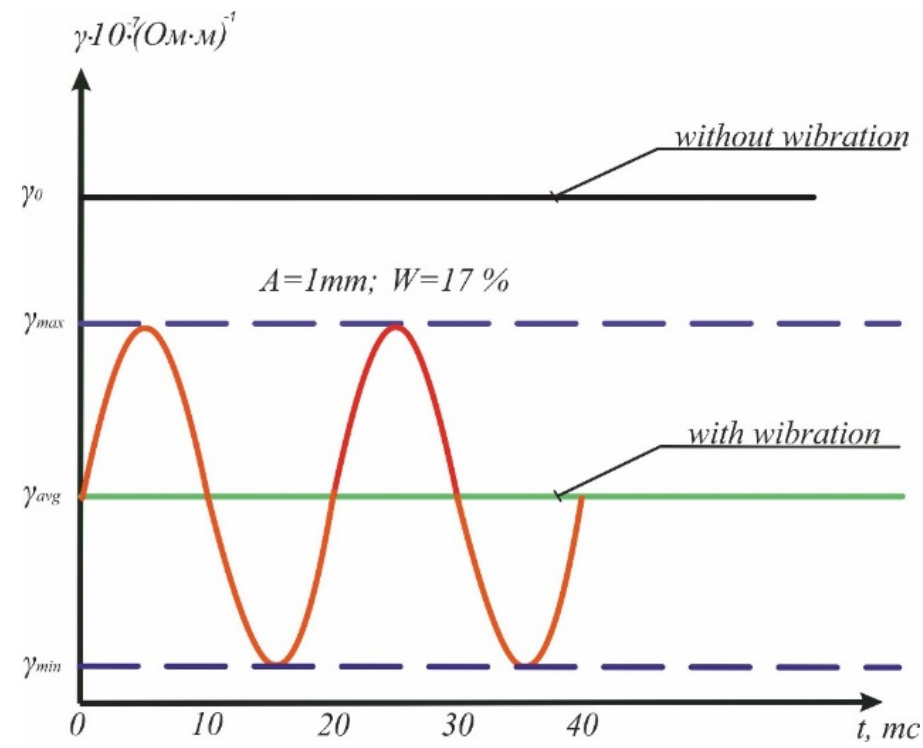

Fig. 1. Oscillogram of instantaneous values of the reach-through conductivity of wheat

"Bezostaya -1" without vibration and under it, by the amplitude $A=1 \mathrm{~mm}$ at moisture of $W=17 \%$

From the resistor 3, the control voltage is supplied to the input of the secondarymeasuring converter, made according to any classical circuit (self-generating, resonant, bridge, etc.), which includes a high-frequency generator and a measuring converter. The device 9 is connected to the output of the converter 8. By changing the position of the resistor 3 engine, it is possible (ceteris paribus) to change the voltage value $U_{\text {thres. }}$, applied to the input key 7 , and thereby change the time intervals $t_{1}-t_{2}, t_{3}-t_{4}$ etc., during which the key is open and the dielectric characteristics $\varepsilon$ of bulk material are recorded.

Timing diagrams of conformity shown in Fig. 3 illustrate the operation of the device. The inductance of the coil of the electromagnetic vibrator 2 leads to a phase shift between the current $I_{e m}$ in the coil and the half-wave voltage of the power source 1. The switchingon of the resistor 3 in series with the

From the diagrams in Fig. 3 it can be seen that the driving force of vibration $F$ is in phase with voltage $U_{\text {con. }}$ which controls the operation of the key, while the maximum value of the force $F$ is achieved with a minimum air gap of the vibrator's magnetic system. With the minimum values of the air gap of the vibrator's magnetic system, the value of the reach-through conductivity $\gamma$ reaches the minimum values. At these moments, the voltage $U_{\text {con }}$ from the resistor 3 is fed to the input of the threshold key 7 . When the voltage at the key input is equal to the threshold of action $\left(U_{\text {thres. } k}\right)$ its input signal $\left(U_{\text {out.k }}\right)$ (Fig. 3), during the time intervals $t_{1}-t_{2}, t_{3}-t_{4}$ etc., is fed to the controlled input of the high- 
frequency converter8 and starts it up for the indicated time intervals, that is, when the converter is launched, the moisture (capacity) of the bulk material is recorded.

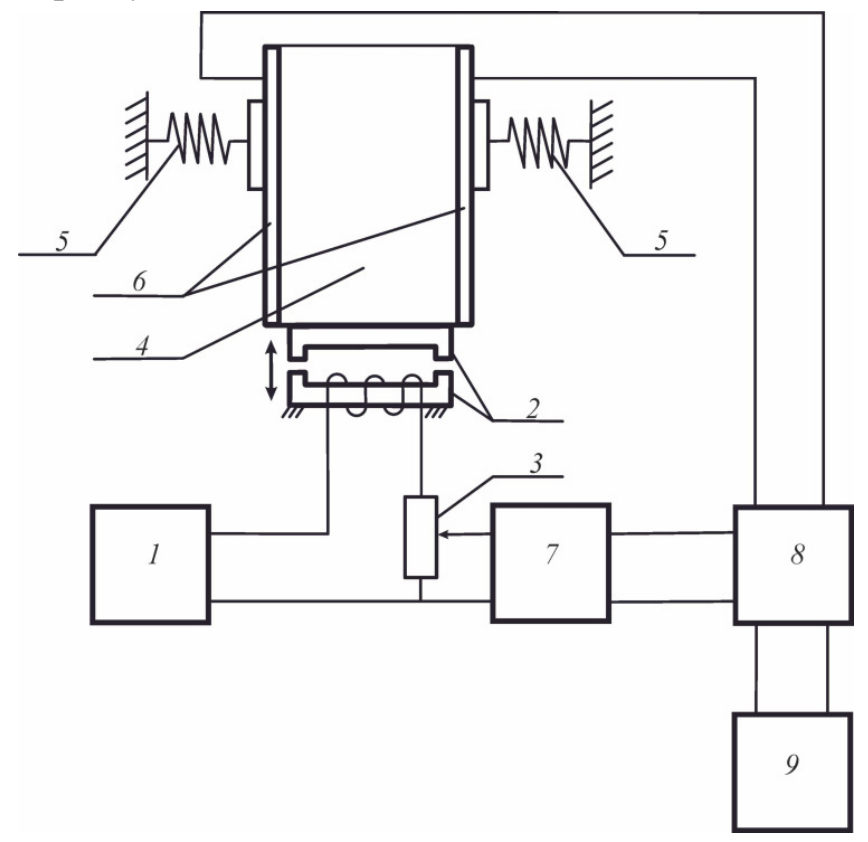

Fig. 2. Diagram of the device that implements the method of measuring the electrical permeability of grain mass: 1 - half wave voltage source; 2 - electromagnetic vibrator; 3 - resistor; 4 - capacitive sensor; 5-springs; 6 - capacitor plates; 7 - threshold key; 8 - secondary converter;

9 - measuring device, coil of the vibrator 2 allows to control the current key 8 , as the current $I_{e m .}$ of the coil and control voltage $U_{c o n .}$ are in phase

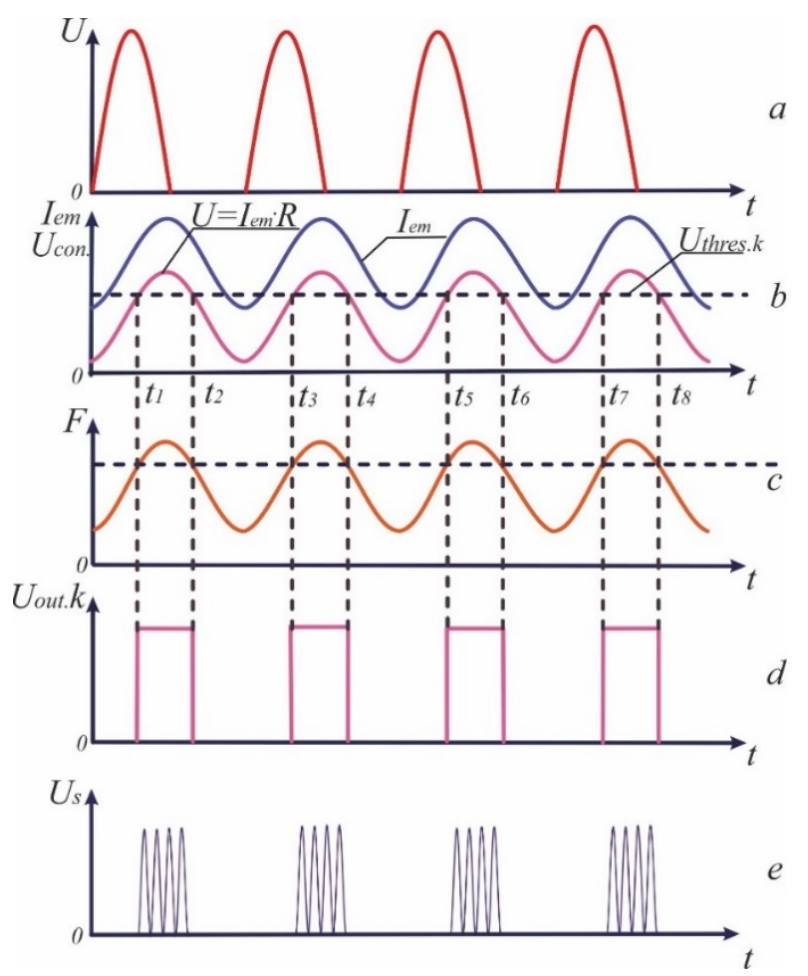

Fig. 3. Timing diagrams of conformity: $a$ - voltage $U ; b$ - electromagnetic vibrator current $I_{e m}$ and key control voltages $U_{\text {con. }} ; c$ - vibration driving force $F ; d$ - output key voltage $U_{\text {out.k. }}$; $e$ - packs of high frequency voltage $U_{s}$, entering the sensor

In this case, as it can be seen in Fig. 2, on the plates of the 6-capacitive sensor from the converter 8 packs of high-frequency voltage $U_{s}$ is fed with duration $t_{1}-t_{2}, t_{3}-t_{4}$ etc. The duration of these time 
intervals is determined by the threshold of the key reaction 7and can be adjusted (see Fig. 2) by the resistor 3 . Thus, moisture is measured at lower values of the reach-through conductivity compared to its average value (Fig. 1).

The method described above is original and allows measurements at intervals of exposure to the driving force of the vibrator corresponding to the minimum value of the reach-through conductivity.

\section{Results and discussion}

For the purpose of moisture control, a nonlinear-capacitive measuring converterdiagram has been selected, a feature of which is the presence of a low-frequency switcher $S$ (acting as a modulator), controlled from a low-frequency generator $(L F G)$ of an alternating voltage (Fig. 4).

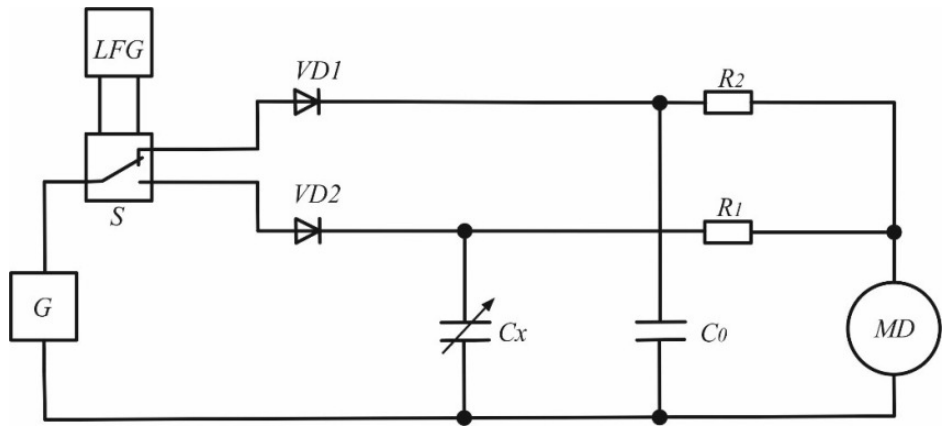

Fig. 4. Non-linear capacitive measuring converter diagram

This non-linear capacitive measuring converter (CMC) operates as follows. Generator $G$ generates unipolar rectangular pulses of high frequency (Fig. 4); the low-frequency switcher $S$ alternately connects to the output of the generator $G$ diodes $V D_{1}$ and $V D_{2}$, connected by the same electrodes to the outputs of the switcher, through which the capacitors $C_{x}$ and $C_{o}$ are charged.

The state diagrams of the outputs of the switcher are shown in Fig. $5 b$ and $c$.

In the time interval $t_{1}-t_{2}$ the diode $V D_{1}$ is connected to the generator Gand the capacitor $C_{x}$ is charged; in the time interval $t_{2}-t_{3}$ the diode $V D_{2}$ is connected to the generator and the capacitor $C_{o}$ is charged; in the time interval $t_{3}-t_{4}$ - again the diode $V D_{1}$ and etc. in the indicated sequence with the frequency of the control voltage from the low-frequency source $L F G$.

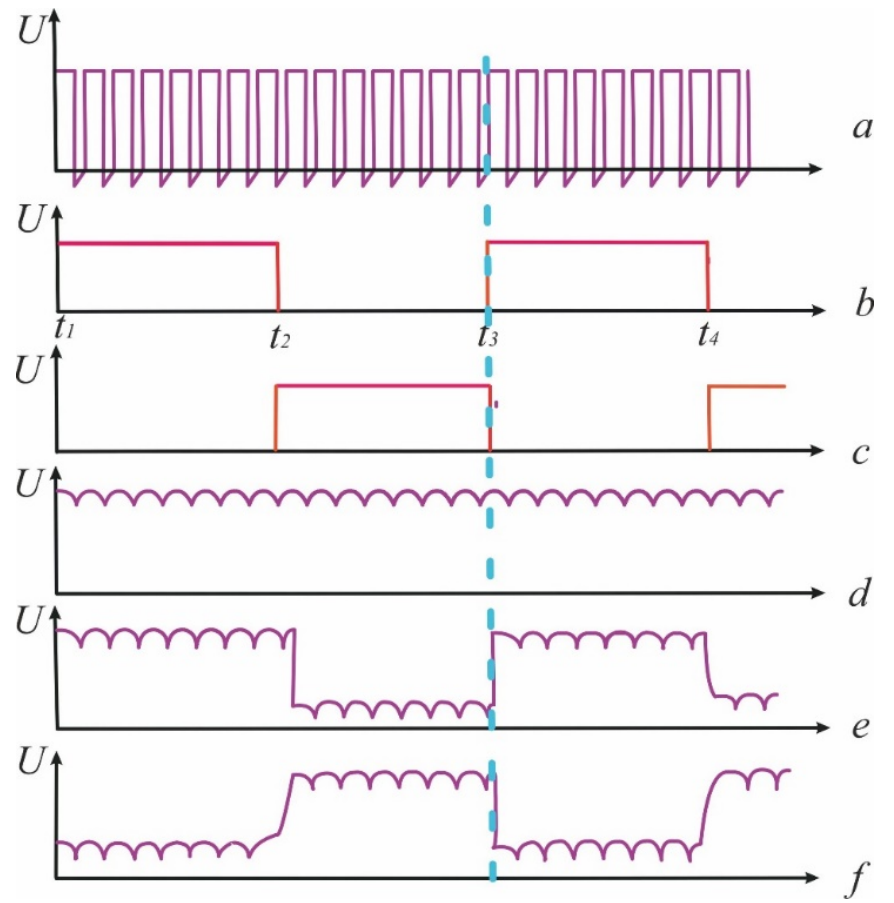

Fig. 5. Generator input voltage diagrams $(a)$; at the output of the switcher $(b, c)$; at the output of the $\operatorname{EMD}(d, e, f)$ 
With such an inclusion of diodes, which is possible due to the use of a switcher, the capacitors $C_{x}$ and $C_{0}$ charge under identical conditions, even in the case of alternating voltage generators of obviously asymmetric form. The discharge of the capacitors $C_{x}$ and $C_{o}$ occurs through the corresponding resistors $R_{1}$, and $R_{2}$ and the input circuit of the measuring device $M D$. If the capacitors $C_{x}-C_{0}$ are equal (and ceteris paribus) each of them will be charged to the same voltage level, and only a direct current will flow through the input circuit of the measuring device $M D$ (Fig. $5 d$ ), to which the device is insensitive.

High-frequency pulsations, if they interfere with changes, can be easily filtered by known means.

If the capacitors $C_{x}$ and $C_{o}$ are equal, for example, for $C_{X}>C_{0}$, the capacitor of the converter $C_{x}$ will be charged to a higher voltage than the capacitor $C_{o}$. This will lead to an increase in the discharge current $C_{x}$, flowing at time instants $t_{1}-t_{2}, t_{3}-t_{4}$ etc. (Fig. 5 e) in comparison with the discharge capacitor $C_{o}$, flowing at alternating times $t_{1}-t_{2}, t_{3}-t_{4}$ etc. A low-frequency alternating component of the discharge current will appear in the input circuit of the measuring device $M D$, the value of which can be used to judge the capacitance of the converter $C_{x}$.

If the capacitor $C_{x}$ becomes smaller than the capacitor $C_{o}$, then the alternating component of the discharge current will change the phase by $180^{\circ}$ (Fig. $5 f$ ).

\section{Conclusions}

1. Since the charge of the capacitors $C_{x}$ and $C_{o}$, in the considered EMD occurs by unipolar pulses, it is possible to use high-frequency unipolar generators with any unspecifiedpulse shape in this case. Moreover, the shape of the pulses does not affect the measurement results, which facilitates the task of selecting and designing high-frequency generators.

2. High-frequency pulsed unipolar generators have lower internal resistance compared to harmonic signal generators, which in turn leads to an increase in sensitivity.

3. When constructing self-compensating measuring circuits, phase-sensitive AC amplifiers are often used as a zero indicator, which differ from DC amplifiers in higher metrological characteristics. Therefore, in measuring circuits with an output signal in the form of a direct current, lowfrequency modulators are used.

4. The resistance value of a contactless switcher in a closed state for germanium semiconductors is several Ohms, for silicon semiconductors several tens of Ohms. As a result, when using contactless switchers, the sensitivity of the circuit decreases sharply. But, despite this, the use of contactless switchers is beneficial in terms of size and reliability.

In addition, the use of switchers can further simplify the circuit and improve measurement accuracy.

\section{References}

[1] Nelson S. O. dielectric properties of agricultural products and some of their applications. To solve. AGR. Eng., 54, 2008, pp. 104-112.

[2] Nelson S.Dielectric Properties of Agricultural Materials and Their Applications. Academic Press, 2015. 229 p.

[3] Nelson S. O., Trabelsi S., Lewis M. A. Microwave sensing of moisture content and bulk density in flowing grain and seed. Transactions of the ASABE 59(2), 2016, pp. 429-433. DOI: $10.13031 /$ trans.59.11377.

[4] Kent M. Electrical and dielectric properties of food materials: a bibliography and data. Science and Technology Publishers Ltd, 1987, 149 p. ISBN 0948323604.

[5] Arslan S., F. Inanc J., Gray N., Colvin T. S. Grain flow measurements with X-ray techniques. Computers and Electronics in Agriculture 26, 2000, pp. 65-80. DOI: 10.1016/S01681699(00)00074-0.

[6] Ranjbaran M., Zare D. Simulation of energetic- and exergetic performance of microwave-assisted fluidized bed drying of soybeans, Energy Volume 59, 15 September 2013, pp. 484-493. DOI: 10.1016/j.energy.2013.06.057.

[7] Stetson L.E., Nelson S.O. Audiofrequency dielectric properties of grain and seed // Transaction of Engineering Research, 1981. vol. 26(2), pp. 171-178. 
[8] Azadi M.S., Younesi E. The effects of storage on germination characteristics and enzyme activity of sorghum seeds. Journal of Stress Physiology \& Biochemistry, Vol. 9(4), 2013, pp.289-298.

[9] Khairetdinova A.F., Saitov R.I., Abdeev R.G., Talipov N.S. A study of a hygrothermal method of grain moisture control in the drying process. Measurement techniques, Vol. 55(1), 2012, pp. 104107.

[10] Kok Y.Y., Hou K.M., Li L.Y., Jamaliah S., Zulkifly A. A Small and Slim Coaxial Probe for Single Rice Grain Moisture Sensing. Sensors, Vol. 13 (3), 2013, pp. 3652-3663.

[11] Meszaros P. Relationships between electrical parameters and physical properties of cereal grains, oilseeds, and apples PhD diss. Budapest, 2007.

[12] Ivashina A., Adoshev F., Zhdanov V., Shemyakin V. Studying the electrical characteristics of grain under vibration action. Engineering for Rural Development. - Jelgava, 2019, pp. 613-618. 